\title{
ApplicationForum \\ Pitfalls of Protein Extraction by RIPA Buffer
}

\section{Quanzhi Li Ph.D., Invent biotechnologies Inc}

\section{Introduction}

Protein extraction using RIPA buffer is a common practice. However due to the lack of comparable method, there is no direct comparison of protein profiles extracted by RIPA buffer and other methods. Here we report a side by side comparison between a rapid (1- $3 \mathrm{~min}$ ) novel protein extraction kit and RIPA buffer.

\section{Materials and Methods}

Proteins were extracted from mouse liver and splenocytes by Minute ${ }^{\mathrm{TM}}$ total protein extraction kit for animal cultured cells/tissues (referred to as Minute kit below) and RIPA buffer (R0278, Sigma). Briefly, samples using Minute kit were prepared according to manufacturer's instruction. There is no insoluble fraction with Minute kit. For RIPA buffer, tissues were homogenized and extracted for 30 min with shaking. The lysates were centrifuged at 12,000xg for $10 \mathrm{~min}$. The residual insoluble fraction of RIPA extraction was further extracted using the Minute kit and analyzed by $12 \%$ SDS-PAGE.

\section{Results and Discussion}

A side by side protein profile comparison is shown in Figure 1. The protein profiles of the two methods are similar but not identical.

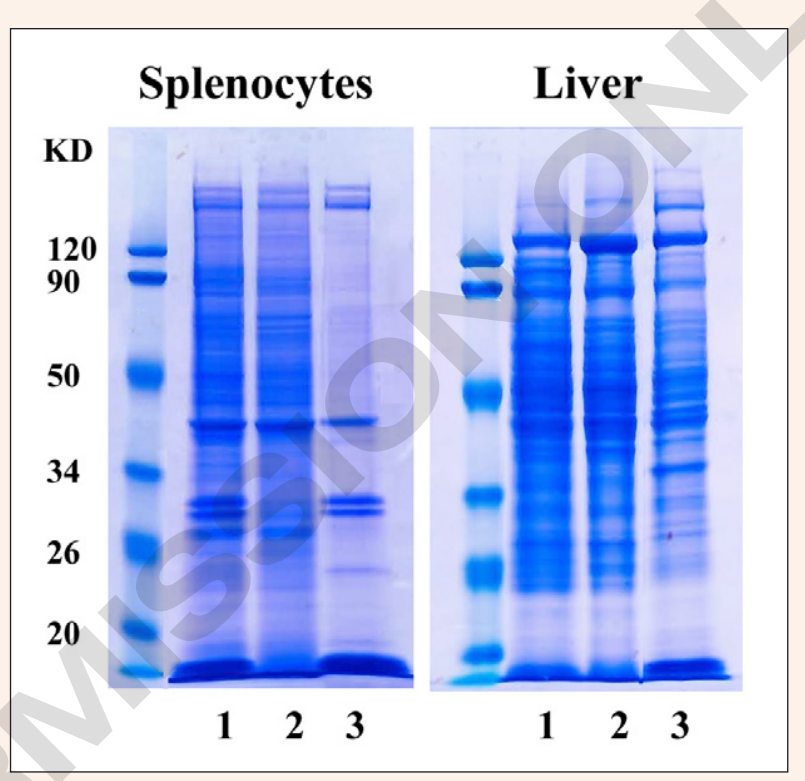

Figure 1. Comparison of protein profiles extracted by Minute kit and RIPA buffer. Lanes 1, 2 and 3 are proteins extracted by Minute kit, RIPA buffer and insoluble fraction of RIPA extraction respectively.

Proteins extracted by RIPA buffer contain significant less proteins in low molecular weight area (lane 2) but they are significantly increased in residual insoluble fraction (Lane 3). Large numbers of protein are found in post-RIPA extraction fractions and the protein profiles in Lanes 2 and 3 are not the same, indicating that significant amount of protein is lost to the insoluble fraction and that the protein loss is non-proportional and somewhat selective. The profiles of lost proteins are obviously different in the two samples. The size distribution of lost proteins covers the whole protein spectrum ranging from $>120$ to $<20 \mathrm{KDa}$. Ngoka (1) compared the protein profiles of RIPA extracted proteins and proteins extracted from the insoluble fractions of breast cancer tissues by mass spectrometry and found that nearly all extracellular matrix (EMC) and many other proteins are exclusively found in the insoluble fractions. EMC proteins are very critical for cancer research (invasiveness and metastasis). Fibronectin was found poorly solubilized in RIPA buffer (2) and altered biochemical pathways with RIPA buffer has also been reported (3). Based on these findings, it is obvious that the protein profile extracted by RIPA buffer is incomplete and altered. Serious data interpretation issues could arise for many qualitative and quantitative experiments using RIPA buffer only. Data verification by other methods should be considered. In view of the fact that Minute kit can extract proteins from post-RIPA extraction insoluble fraction efficiently, it is superior to RIPA buffer in terms of completeness of protein profile, ease of use, speed and performance. For more info visit: inventbiotech.com

\section{References}

1. Lamber CM Ngoka (2008). Proteome Science. 6:1 (1).

2. Grover A. and Admson ED. (1985). J Biol Chem 260:12252-12258

3. Deseau et al., (1987). J Cellular Biochem 1987, 35:113-128. 